\title{
Initial distribution of the volatile-rich asteroids inferred from the isotopic records: Implication for return samples from Ryugu
}

\author{
RYOTA FUKAI ${ }^{1}$ AND SOTA ARAKAWA ${ }^{2}$
}

${ }^{1}$ Japan Aerospace Exploration Agency

${ }^{2}$ National Astronomical Observatory of Japan

Presenting Author: ryota.fukai@gmail.com

Describing the comprehensive evolutionary scenario for asteroids is key to explaining the various physical processes of the solar system. Bulk-scale carbonaceous chondrites (CCs) possibly record the primordial information associated with the formation processes of their parent bodies. In this study, we tried to estimate the relative formation region of volatile-rich asteroids by utilizing the nucleosynthetic $\mathrm{Cr}$ isotopic variation $\left({ }^{54} \mathrm{Cr} /{ }^{52} \mathrm{Cr}\right)$ in bulk-scale CCs. Numerical calculations were conducted to track the temporal evolution of isotopically different (solar and presolar) dust and ${ }^{54} \mathrm{Cr} /{ }^{52} \mathrm{Cr}$ values for mixed materials with disk radius. First, we found that isotopic heterogeneities in $\mathrm{CC}$ formation regions would be preserved with a weak turbulence setting that would increase the timescales of the advection and diffusion in the disk. Second, we assessed the effects of gaps formed by giant planets. Finally, the distance from the injected supernovae and $\mathrm{Cr}$ isotopic compositions of the presolar grains were investigated in terms of the estimated formation region of CCs. In our results, a plausible formation region of four types of CCs can be obtained with the supernova from approximately 2 pc and typical $\mathrm{Cr}$ isotopic compositions of presolar grains. We speculate that the heterogeneities in the other types of supernovaderived nuclides such as ${ }^{50} \mathrm{Ti}$ and ${ }^{62} \mathrm{Ni}$ can be explained by the similar mechanism.

Among the volatile-rich asteroids associated with investigated $\mathrm{CCs}$, the obtained relative orbits from these asteroids are $\mathrm{K}(-$ type) $<\mathrm{Ch}<\mathrm{D}<\mathrm{B}$. This sequence is consistent with the individual model of Desch et al. [1]. However, these results are inconsistent with the present orbits implied from the orbits of their asteroid families $\mathrm{K}<\mathrm{Ch}<\mathrm{B}<\mathrm{D}$ [2]. We speculate that the orbital changes were caused by the migration of Jupiter and Saturn. Similarly, the initial location of Ryugu parent bodies can be assessed by the initial analysis of return samples.

[1] Desch, Kalyaan \& Alexander (2018) The Astrophysical Journal Supplement Series, 238, 11.

[2] Burbine (2014) Treatise on Geochemistry 2nd edition, 365. 